\title{
Identification of Genetic Loci for Sugarcane Leaf Angle at Different Developmental Stages by Genome-Wide Association Study
}

\author{
Xinglong Chen \\ Guangdong Academy of Sciences \\ Zhenghui Huang \\ Zhongkai University of Agriculture and Engineering \\ Xiangbo Zhang \\ Guangdong Academy of Sciences \\ Xiaomin Feng \\ Guangdong Academy of Sciences \\ Jinfang Xie \\ Zhongkai University of Agriculture and Engineering \\ Bin Wu \\ Zhongkai University of Agriculture and Engineering \\ Yiji Luo \\ Zhongkai University of Agriculture and Engineering \\ Mingfeng Zhu \\ Zhongkai University of Agriculture and Engineering \\ Yongwen Qi ( $\square$ yongwen2001@126.com) \\ Guangdong Academy of Sciences
}

\section{Research}

Keywords: Sugarcane, Leaf angle, GWAS, SNPs, Candidate genes

Posted Date: November 1st, 2021

DOl: https://doi.org/10.21203/rs.3.rs-964989/v1

License: (c) (1) This work is licensed under a Creative Commons Attribution 4.0 International License. Read Full License 


\section{Abstract \\ Background}

Sugarcane (Saccharum spp.) is an efficient crop mainly used for sugar and bioethanol production. High yield and high sucrose of sugarcane is always the fundamental demands in sugarcane growth worldwide. Leaf angle and size of sugarcane can be attributed to planting density, which was associated with yield. In this study, we performed Genome-Wide Association Studies (GWAS) in a panel of 216 sugarcane core parents and their derived lines (natural population) to determine the genetic basis of leaf angle and key candidate genes at seedling, elongation and maturity stage.

\section{Results}

A total of 288 significant associated loci of sugarcane leaf angle at different developmental Stages were identified by GWAS. Among them, one key locus and eleven loci was identified in all three stages and two stages, respectively. Overall, 4089 genes were located in the confidence interval of significant loci, among which 3892 genes were functionally annotated. Finally, thirteen core parents and their derivatives tagged with SNPs were selected for Marker-assisted selection (MAS).

\section{Conclusion}

These candidate genes are mainly related to MYB transcription factors, auxin response factors, serine/threonine-protein kinases, etc. The are directly or indirectly associated with leaf angle in sugarcane. This research provided a large number of novel genetic resources for the improvement of leaf angles and simultaneously to high yield and high bioethanol production.

\section{Background}

Sugarcane (sacharum spp.) is the major raw material for the global sucrose supply and the preeminent energy crop for bioethanol production [1]. High biomass yield and high sucrose content are fundamental demands for sugarcane production. Plant architecture is one of the most important character determining the yield of plant. Donald first proposed the concept of ideal plant architecture in 1968 [2]. It is necessary to find the plant architecture that confers the least competition among individuals in the field, which can maximize the utilization of light energy and increase the plant yield. Leaf angle is an important component of sugarcane plant architecture as it can determine how light is acquired and the spatial distribution of the leaves. When the leaf angle is narrow, the blade is vertical oriented, and the plant architecture is compact. While the leaf angle is large, the blade is loose level, and the plant architecture is compact. When the leaf angle is large, the blade is horizontally oriented. Thus, leaf angle can be optimized to reasonably improve the planting density and photosynthetic efficiency of sugarcane, which is an effective method to increase the crop yield. This has been widely used in rice, maize, wheat, and other crops [3-5]. However, the related genes / QTL controlling leaf angle in sugarcane have been rarely reported, mainly due to the complex genetic background of sugarcane. It is helpful to cultivate new varieties with small leaf angles and optimal plant architecture by understanding the characteristics of leaf angles and mining excellent alleles.

The long growth cycle and particular flowering conditions of sugarcane necessitate that "centralized hybridization and regional breeding" is the dominant global sugarcane breeding mode. Specifically, national sugarcane hybrid seed production is undertaken at unified hybrid bases and then the seeds are distributed to the national breeding units, each of which carries out regional sowing and breeds new varieties. Therefore, national sugarcane breeding can only rely on the parents protected by the cross bases. Only a select few parental sources are used for cross breeding every year due to ecological factors and the available human and material resources at the cross base. There are more than 2400 sugarcane germplasm resources in China, but only about 300 of them have been used in crossbreeding. Many excellent varieties, such as Guitang 42, Guiliu 05136 and Yuetang 09-13, have been bred by using these core parents. These core germplasm resources are not only rich in genetic diversity, but also have been subjected to a variety of stresses for natural selection in a long-term evolutionary process, and are thus suitable for cultivation in many regions. These germplasm resources are the natural gene pool for the genetic improvement of cultivated species.

Although sugarcane is aneuploid heteropolyploid and contains a large genome and complex genetic background, with the rapid development of sequencing technology and the high-density SNP markers, Genome-Wide Association Study (GWAS) has become the most powerful method to explore the quantitative characteristics of sugarcane. Furthermore, GWAS has been instrumental in important breakthroughs in yield-related traits, sugar content, and fiber fraction in sugarcane [6-9]. For the complex trait of leaf angle, GWAS should be suitable to identify loci that contribute to this trait. The objective of this study is (i) to figure out the distribution of leaf angle in this panel of 216 sugarcane core parents and their derived lines (natural population) at the seedling, elongation, and mature stage; (ii) to identify the loci significantly associated with leave angle by GWAS; and (iii) to identify possible candidate genes by annotating these loci, which could provide genetic resources useful for the improvement of leaf angle and Marker-assisted selection (MAS) in sugarcane.

\section{Results}

\section{Phenotypic analysis of leaf angle in sugarcane natural population}

The leaf angle varied greatly among different sugarcane accessions in the panel of 216 sugarcane core parents and their derived lines (Fig. 1; Table S1). Leaf angle varied from $12.72^{\circ}$ to $58.20^{\circ}$ in different stages. The average angle of +2 leaves in the seedling stage, elongation stage, and maturity stage was $28.69^{\circ}$, $20.21^{\circ}$, and $21.82^{\circ}$ respectively. While the average angle of +3 leaves were $35.12^{\circ}, 25.36^{\circ}$, and $25.86^{\circ}$, respectively, and the average angle of +4 leaves at 
elongation and maturity were $31.44^{\circ}$ and $30.44^{\circ}$, respectively. Although the average angle at the seedling stage was the widest, the coefficient of variation was low, which indicated that there was little difference in leaf angle among different accessions at the seedling stage. With the growth of sugarcane, the difference of leaf angle became wider, until it reached its widest in the mature stage. The dispersion of leaf angle also increased as the growth of sugarcane with a variation coefficient ranging from 13.78 to 23.78 . Skewness and kurtosis ranged from 0.49 to 1.16 and from 2.82 to 4.70 , respectively. (Table 1 ). As shown in Figure 2, the frequency distribution was a continuous normal distribution or skewed distribution, indicating that sugarcane leaf angle is a quantitative trait controlled by multiple genes. The Pearson's correlation analysis showed that there was a significant positive correlation between leaf angle in each growth stage with a correlation coefficient of $0.44-0.63$, and a high positive correlation among leaves in the same stage with a correlation coefficient of 0.83-0.94 (Fig. 3).

Table 1

Changes of leaf angle at different growth stages of sugarcane

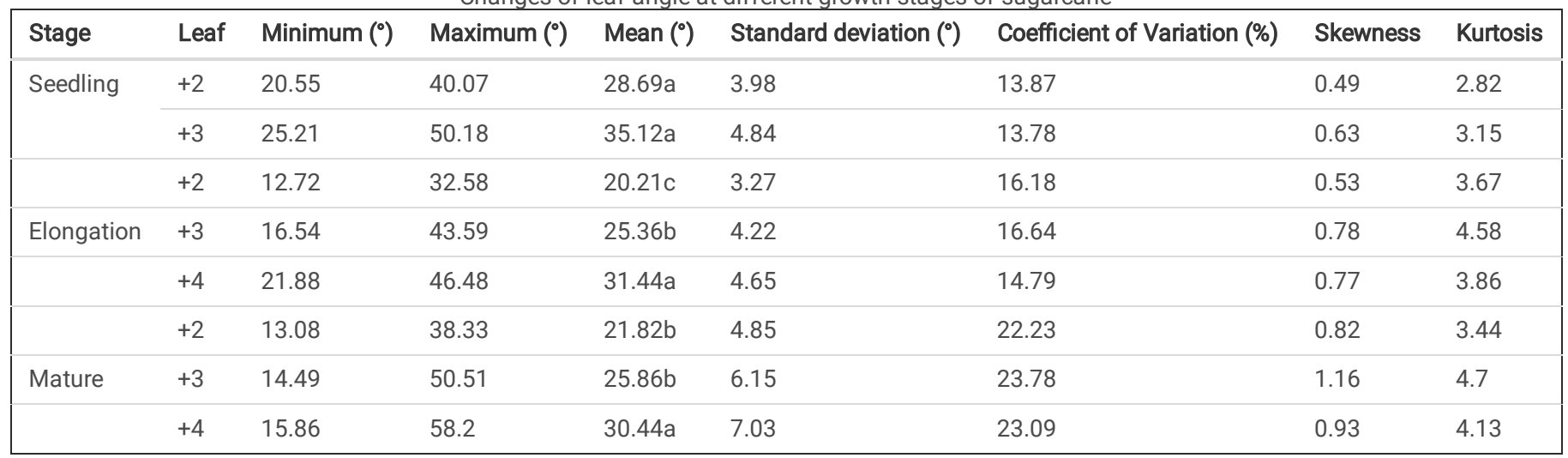

\section{Snp Markers And Population Structure Of The Natural Population}

A total of 4,584,312 SNPs were obtained following filtration and screening with Plink. Among them, 269,523 SNPs (5.88\%) were located within the gene, 144,189 SNPs (3.14\%) were situated upstream of the gene, while 143,302 SNPs (3.12\%) were situated downstream of the gene, and the remaining 4,027,298 SNPs (87.86\%) were located in intergenic regions. Based on the 4027298 high-quality SNP markers, 216 core parents and their derivatives were divided into ten subgroups according to the best K value, they were Africa, Australian, Brazil, China, Cuba, India, Mauritius, Other, Philippines, Taiwan, and USA, respectively, suggesting that our panel may originated from the admixture of ten populations (Fig. 4). Whole-genome SNP markers were initially used to analyze the LD level of sugarcane leaf angle at different growth stages. The correlation coefficient $\left(r^{2}\right)$ was greater than 0.1 , and when $r^{2}$ was 0.10 , the LD decay rate was 10 kb.

\section{Genome-wide Association Study Of Leaf Angle}

A total of 288 SNP loci were found to be significantly associated $(P<0.001)$ with 16 leaf angle phenotypes (Fig. 5, table S2). There were 69,113 , and 119 loci detected in the seedling, elongation, and maturity stage, respectively. One locus (Ss6A_102766953) was mapped in chromosome 6A near the SNP marker of Chr21_102766953 by the phenotype of all these three stages synchronously and accounted for $1.67 \%$ (1.15 to $1.99 \%$ ) of the phenotypic variation (Table 2). Eleven loci were detected by two of these three stages, they were Ss1A_68079563, Ss1A_ 70216053, Ss1A_ 71163098, Ss4A_ 51223571, Ss5A_ 63761550, Ss5C_ 86542573, Ss5D_ 30890024, Ss6A_ 53870145, Ss6C_ 46060170, Ss7C_ 58432083, and Ss7D_67376640, accounting for 1.48-4.90\% of the phenotypic variation (Table 2), and they were considered to be the elite alleles in this study. Furthermore, 14 loci were identified by the +2 and +3 leaves in the seedling stage, while there were three loci detected in the $+2,+3$, and +4 leaves in elongation and mature stages, respectively (Table S2). 
Table 2

List of candidate genes associated with elite loci of sugarcane leaf angle

\begin{tabular}{|lllll|}
\hline SNP tag & Candidate gene & E-value $^{\mathrm{a}}$ & PVE(\%) & Functional annotation \\
\hline Ss1A_68079563 & Sspon.01G0018220-1A & $2.35 \mathrm{E}-06$ & 2.92 & Sulfate transporter \\
\hline Ss1A_70216053 & Sspon.01G0018870-1A & $3.00 \mathrm{E}-06$ & 1.74 & Ubiquitin-conjugating enzyme \\
\hline Ss1A_71163098 & Sspon.01G0019040-1A & $2.49 \mathrm{E}-06$ & 2.21 & Auxin response factor \\
\hline Ss4A_51223571 & Sspon.05G0009970-2D & $1.32 \mathrm{E}-06$ & 1.84 & amino acid transporter ANTL1-likeG \\
\hline Ss5A_63761550 & Sspon.05G0015920-1A & $1.32 \mathrm{E}-06$ & 1.48 & Serine/threonine-protein kinase \\
\hline Ss5C_86542573 & Sspon.05G0036390-1C & $4.54 \mathrm{E}-07$ & 1.49 & Transcription factor MYB82-like isoform X1 \\
\hline Ss5D_30890024 & Sspon.05G0009970-2D & $1.28 \mathrm{E}-06$ & 4.78 & amino acid transporter ANTL1-like \\
\hline Ss6A_53870145 & Sspon.06G0009970-1A & $1.35 \mathrm{E}-06$ & 4.90 & Glucose-6-phosphate/phosphate-translocator precursor \\
\hline Ss6A_102766953 & Sspon.06G0018820-1A & $1.43 \mathrm{E}-06$ & 1.67 & MYB transcription factor \\
\hline Ss6C_46060170 & Sspon.06G0010540-2C & $2.24 \mathrm{E}-06$ & 2.86 & Serine-rich protein-related \\
\hline Ss7C_58432083 & Sspon.07G0033260-1C & $7.95 \mathrm{E}-07$ & 2.78 & Thylakoid membrane protein TERC chloroplastic \\
\hline Ss7D_67815942 & Sspon.07G0034190-2D & $1.55 E-06$ & 3.13 & Probable serine/threonine-protein kinase \\
\hline a Indicates the significantly associated SNP sequences blast with transcriptome library of sugarcane based on E-value < 10-5. \\
\hline b Indicates the phenotypic variation explained by the mean value of the individual locus detected in different stages.
\end{tabular}

\section{Candidate Gene Analysis}

Candidate genes were searched in the range of LD decay distance (500 kb) of SNP upstream and downstream of -log10 ( $P$-value) within each locus. According to the annotation of gene function and its expression position/expression level in the reference genome, the most likely candidate gene was selected as the candidate gene of this site. A total of 5571 candidate genes were located in these 288 loci, of which 1838 had GO functional annotations (Table S2). These candidate genes are mainly related to brassinosteroid LRR receptor kinase precursor, auxin response factor, gibberellin receptor, chloroplastic, auxin synthesis/ signal transduction, Serine / threonine-protein kinase, and various transcription factors. They are directly or indirectly associated with leaf angle.

\section{Core parents and their derivatives with narrow leaf angle tagged with SNPs}

A total of 13 germplasm resources carrying a different combination of elite loci and narrow leaf angle $\left(<30^{\circ}\right)$ in all these stages were selected in Table 3 . Marker heterozygosity remained in all the core parents and their derivatives except for Xuan-15, such as \#1626 at Snp8 (Ss6A_102766953) and Snp12 (Ss7D_67815942), CP57-614 at Snp7 (Ss5D_ 30890024) and Snp9 (Ss6A_ 53870145), CP89-2143 at Snp5 (Ss5A_63761550) and Snp9 (Ss6A_ 53870145), etc. This suggested that these materials can also be used for fine mapping of these loci. These 13 core parents and their derivatives with their leaf angle and nearest marker should be useful for improving sugarcane leaf angle via marker-assisted selection (MAS). 
Table 3

The elite core parents and their derivatives with their small leaf angle and nearest markers.

\begin{tabular}{|c|c|c|c|c|c|c|c|c|c|c|c|c|c|c|c|c|}
\hline Name & S2+ & S3+ & E2+ & E3+ & E4+ & M2+ & M3+ & M4+ & Snp1 & Snp2 & Snp3 & Snp4 & Snp5 & Snp6 & Snp7 & Snp8 \\
\hline 1626 & 25.29 & 29.01 & 15.39 & 20.51 & 28.55 & 13.95 & 17.80 & 20.78 & $\mathrm{~T}$ & A & C & G & C & C & G & C \\
\hline СР57-614 & 22.01 & 29.55 & 14.41 & 17.09 & 22.59 & 15.65 & 16.92 & 20.00 & $\mathrm{~T}$ & G & C & $\mathrm{T}$ & C & C & G & $\mathrm{G} / \mathrm{C}^{\mathrm{b}}$ \\
\hline CP89-2143 & 24.62 & 28.29 & 18.75 & 22.29 & 28.84 & 18.17 & 20.59 & 24.57 & $\mathrm{~T}$ & G & C & $\mathrm{T}$ & $\mathrm{C} / \mathrm{T}^{\mathrm{b}}$ & $\mathrm{T}$ & A & C \\
\hline GT-03-411 & 24.22 & 29.88 & 18.24 & 22.27 & 25.23 & 18.47 & 21.25 & 25.35 & $\mathrm{~T}$ & G & C & $\mathrm{T} / \mathrm{G}^{\mathrm{b}}$ & C & C & G & C \\
\hline $\begin{array}{l}\text { HOCP03- } \\
708\end{array}$ & 21.66 & 29.06 & 14.89 & 19.10 & 27.59 & 17.00 & 20.71 & 23.56 & $\mathrm{~T}$ & A & C & $\mathrm{T}$ & C & C & G & C \\
\hline $\begin{array}{l}\text { Liucheng05- } \\
291\end{array}$ & 21.48 & 28.42 & 17.75 & 22.00 & 26.73 & 15.49 & 18.79 & 20.80 & $\mathrm{~T}$ & $\mathrm{G} / \mathrm{A}^{\mathrm{b}}$ & C & $\mathrm{T}$ & C & C & G & C \\
\hline $\begin{array}{l}\text { Liucheng06- } \\
241\end{array}$ & 22.77 & 27.88 & 17.82 & 23.06 & 27.83 & 13.08 & 14.49 & 15.86 & $\mathrm{~T}$ & $\mathrm{G} / \mathrm{A}$ & C & $\mathrm{T} / \mathrm{G}$ & C & C & G & C \\
\hline $\begin{array}{l}\text { Neijiang57- } \\
416\end{array}$ & 20.91 & 25.35 & 18.33 & 23.02 & 28.45 & 17.27 & 20.86 & 26.39 & $\mathrm{~T}$ & G & C & $\mathrm{T}$ & C & C & G & C \\
\hline Xuan-15 & 24.12 & 28.19 & 14.98 & 18.53 & 25.61 & 19.05 & 24.16 & 24.66 & $\mathrm{~T}$ & G & C & $\mathrm{T}$ & C & C & G & C \\
\hline YCE07-71 & 23.04 & 27.49 & 15.27 & 19.84 & 27.25 & 21.42 & 24.16 & 29.96 & $\mathrm{~T}$ & A & $\mathrm{C}$ & $\mathrm{T}$ & $\mathrm{C}$ & C & G & C \\
\hline YT-01-71 & 22.75 & 28.54 & 15.99 & 20.84 & 24.81 & 18.92 & 20.74 & 23.14 & $\mathrm{~T}$ & G & C & $\mathrm{T}$ & C & C & G & G \\
\hline Zhanzhe-40 & 25.11 & 28.88 & 12.72 & 16.54 & 21.88 & 15.13 & 16.55 & 19.09 & $\mathrm{~T}$ & G & C & $\mathrm{T}$ & C & C & G & $\mathrm{G} / \mathrm{C}$ \\
\hline Zhanzhe-50 & 20.55 & 26.89 & 16.17 & 19.45 & 22.72 & 16.39 & 18.08 & 20.54 & $\mathrm{~T}$ & G & C & $\mathrm{T}$ & C & C & G & C \\
\hline
\end{tabular}

a $\mathrm{S} 2+$ and $\mathrm{S} 3+$ denotes leaf angle of $+2,+3$ in seedling stage, E2+, E3+, E4+, M2+, M3+ and M4+denotes leaf angle of $+2,+3$, and +4 in elongation and mature were the nearest markers of Ss1A_ 68079563, Ss1A_ 70216053, Ss1A_ 71163098, Ss4A_ 51223571, Ss5A_ 63761550, Ss5C_ 86542573, Ss5D_ 30890024, Ss Ss6C_ 46060170, Ss7C_ 58432083, Ss7D_67815942, respectively.

$\mathrm{b}$ indicates heterozygous.

\section{Discussion}

The leaf angle of sugarcane is an important factor for determining plant architecture. Compact plant architecture can improve photosynthetic efficiency through reasonable close planting to enhance the yield of sugarcane per unit field area [19-20]. However, the research related to sugarcane plant architecture and leaf angle is focused on epigenetics, maybe due to the polymorphism, high chromosome numbers, and large complex genome size of sugarcane. To study leaf angle deeply, it is necessary to find out the genes/QTLs that determine leaf angle in sugarcane. In this study, 288 SNP loci were found to be significantly associated with leaf angle at the seedling, elongation, and mature stage by GWAS. 69, 113, and 119 loci were detected at the seedling, elongation, and mature stage, respectively. This might be related to the variation in leaf angle at each stage. The average leaf angle at the seedling stage was $31.91^{\circ}$, but the coefficient of variation was low among all the materials. The leaf angle at the elongation and mature stages was $26.04^{\circ}$ and $25.67^{\circ}$ with a wider variation, respectively. Moreover, Pearson's correlation analysis showed that there was a significant positive correlation between leaf angle at each stage, especially for different leaves at the same stage, which was consistent with the GWAS mapping results. Among them, one QTL was identified at all three stages synchronously, which is likely to be a major QTL controlling leaf angle in sugarcane, while the other 11 loci were detected at two stages, indicating that these major QTLs can be stably inherited and are not easily affected by the environment. This is consistent with the conclusions obtained in rice by Hittalmani et al. [21] and Xu et al. [22]. In addition, many identified loci did not overlap across different periods and leaves, which might be due to the influence of different growth stages and environmental factors.

In this study, 12 elite loci that determine sugarcane leaf angle were discovered. Ss6A_102766953 was stably identified from all three stages, and the candidate genes within this locus indicated that MYB transcription factors might play a role in determining leaf angle. The MYB transcription factor family is one of the largest transcription factor families in plants and is involved in the developmental process of plant cell differentiation, morphology, etc. Dubos et al. [23] found that MYB transcription factors regulate Arabidopsis growth and development, auxin response, primary and secondary metabolism, cell fate determination, plant growth and development, and responses to various biotic and abiotic stresses. Zhang et al. [24] and Shin et al. [25] found that MYBs are induced by ABA, IAA, CTK, GA, ethylene, and other plant hormones, indicating that MYB transcription factor genes in plants are widely involved in the responses to plant hormones. Cao et al. [26] found that OS JAMyb encoding the 2R-MYB protein is expressed in root, stem, leaf, leaf sheath, panicle, and other parts of rice. Therefore, it is suggested that this candidate gene might be associated with leaf angle and can be further studied in the future.

Auxin is an important signaling molecule and regulates the growth and development of plants, such as promoting cell elongation, vascular differentiation, regulating the size of leaf angle, etc. In this research, Ss1A_ 71163098 was detected in the seedling and elongation stages, and the candidate genes showed that this locus contains an auxin response factor. Previously Moon et al. [27] found that auxin accumulated at the boundary between the leaf and sheath through fluorescence imaging of the auxin-directed transport protein ZmPIN1a, indicating that auxin is involved in the positional initiation of the leaf sheath Zhang et al. [28] identified a gene that controls leaf angle, $L A Z Y 1$, on maize chromosome 4 via map-based cloning, which showed the change of leaf angle caused by auxin effects on cell development. The auxin-related gene FIB identified in rice is homologous to the auxin biosynthesis gene TAA in Arabidopsis, 
encoding tryptophan aminotransferase. The functional deletion mutants of FIB showed smaller leaves and larger leaf angles [29]. $L r 47$ affects auxin signal transduction by inhibiting the interaction between C-22-hydroxylation and 6-deoxybrassinolide, and controls the curvature of the pulvinus, resulting in larger leaf angles and oblique leaf elongation [30]; The elongation of leaf occipital cells in LC1 mutant plants is affected by auxin and has an increased leaf angle phenotype [31]. The F-box protein TIR1 regulates the angle of rice leaves by binding IAA and Aux / IAA, which leads to ubiquitination, degradation, and release of ARF transcription activity. Overexpression of OsIAA1, which encodes Aux / IAA protein, reduced the inhibition of auxin treatment on root elongation but increased the sensitivity to 24-epibrassinolide in rice. Overexpression of OsIAA1 resulted in significant morphological changes such as decreased plant height and increased leaf angle [32]. In addition, IAA can also participate in the regulation of rice leaf angle through interaction with BR. IAA is involved in the OsBRI1mediated BR signal transduction pathway. OsARF11 and OsARF19 bind and stimulate the promoter of OsBR/1 to induce changes in leaf angle in rice [4, 33].

Some functional kinases, such as serine / threonine protein kinase, are also the main factors regulating leaf angle. Ss $5 A_{\text {_ }} 63761550$ was identified in elongation stage and maturity stage, and the candidate genes showed that this locus is associated to serine/threonine-protein kinase. ILA1 is a functional kinase with serine/ threonine kinase activity, which mainly exists in the nucleus and expresses in the vascular bundles of the leaf pillow. It affects the leaf angle by regulating the formation of mechanical tissue and the abnormality of cell wall composition of the rice leaf pillow.The T-DNA insertion mutant ila 1 showed the character of increased leaf angle. Through the anatomical analysis of ila1 mutant, it was found that the number of vascular bundles in the leaf pillow decreased and the number of thick walled cells decreased. Moreover, the mechanical tissue abnormality of the mutant leaf pillow led to the lower content of cellulose and other cell wall monosaccharides, which led to the poor mechanical support of the mutant and the increased leaf angle [34].

Many other elite loci mapped in this study are also very important in regulating the leaf angle. The candidate gene Ss $1 A \_68079563$ is predicted to be a sulfate transporter $\left(\mathrm{H}^{+} / \mathrm{SO}^{4}{ }_{2-}\right.$ cotransporter). Their transport function depends on the membrane potential gradient maintained by an $\mathrm{H}^{+}$pump, and the sulfate transporter is higher in mature or older leaves [35]. The candidate Ss $1 A_{-} 70216053$ binds ubiquitin-conjugating enzymes, which is mainly involved in the ubiquitin-proteasome system [36], regulating ethylene, GA, IAA, and other hormone signal transduction [37-39], thereby indirectly affecting the leaf angle. The candidate genes Ss4A_ 51223571 and Ss5D_ 308900244 are associated with amino acid transport, which is necessary for the growth and development of plants [40]. The candidate gene Ss6A_ 53870145 is related to the glucose-6-phosphate/phosphate-translocator precursor, which is preferentially expressed in non-green tissues and mediates the transport of glucose-6-phosphate (Glc-6-P), triose phosphate, and glycerol-3-phosphate (3-PGA), Plastids of non-green tissues are the main storage sites of carbohydrates as starch in heterotrophic tissues. Through GPTs, non-green plastids can transfer sugar from the cytoplasm into carbon sources in the form of Glc-6-P to drive the synthesis of important substances such as fatty acids, amino acids, and starch, thus providing precursors for the pentose phosphate pathway (OPPP) [41]. The candidate Ss7C_ 58432083 associates with the thylakoid membrane protein TERC in the chloroplast, and plays an active role in protein transport, photosystem assembly, and thylakoid membrane stability [42]. The candidate genes of these excellent SNPs are directly or indirectly related to leaf angle and should be further investigated in the future.

\section{Conclusion}

In summary, a total of 288 SNP loci that contribute to leaf angle were identified by GWAS at the seedling, elongation, and mature stages of development in sugarcane. Twelve of these SNPs were detected in at least two of three developmental stages. The candidates of these elite loci were analyzed compared to the function of their homologs in rice, corn, Arabidopsis, and other plants in this study. These candidate genes are mainly related to MYB transcription factors, auxin response factors, serine/threonine-protein kinases, sulfate transporters, ubiquitin-conjugating enzymes, amino acid transporters, glucose-6phosphate/phosphate-translocator precursors, and the thylakoid membrane protein TERC. Thirteen core parents and their derivatives tagged with SNPs (Table 3) can be used as narrow-leaf angle donors for MAS.

\section{Methods}

\section{Plant materials and growth}

In this study, a panel of 216 core parents and their derivatives were selected to construct a sugarcane natural population (Table S1). They were derived from many sugarcane planting countries, China (150), United States (32), Australia (10), India (4), Cuba (7), Brazil (2), France (1), Philippines (4), Mauritius (2), South Africa (2), Thailand (1) and Indonesia (1). Due to their excellent performance in yield, disease resistance, sugar content, and other agronomic traits, most of them were selected as breeding parents in China. This group represents an important genetic background in China.

The natural population was planted in Wengyuan base of Institute of Nanfan \& Seed Industry, Guangdong Academy of Sciences (Guangzhou Sugarcane Research Institute) in 2019. The base is located at $24^{\circ} 17^{\prime} 00^{\prime \prime} \mathrm{N}$ and $113^{\circ} 56^{\prime} 25^{\prime \prime} \mathrm{E}$ with an altitude of $120 \mathrm{~m}$. The experimental design consisted of a completely randomized group with two repeats. Practices were used to ensure that seedling emergence was regular and the spacing of each seedling was uniform. Before planting, seedlings were disinfected, cut into single buds, and transplanted to the field after the seedlings grew to $20 \mathrm{~cm}$ at a row spacing of $1.1 \mathrm{~m}$ and a plant spacing of $25 \mathrm{~cm}$. There were three rows of repeat planting and 16 plants in each row. Only 10 plants in the middle of the plots were investigated. The field management was carried out according to conventional field management, with normal fertilization, irrigation, and control of diseases, pests, and weeds.

\section{Phenotype and statistical analysis}

Due to the leaves at the seedling stage being few in number and the +1 leaf being too close to the heart leaf for facile measurement, the leaf angle of the +2 leaf and +3 leaf of each accession was measured at the seedling stage (Roughly two months old) of the natural population. The leaf angle of the +2 leaf, +3 leaf, and +4 leaf of each accession were measured at the elongation and mature stage, respectively. The phenotypic data were analyzed by Excel 2010. 
The genomic DNA of this natural population was extracted according to the method described by [10]. Resequencing was performed by the Beijing Nuohe Zhiyuan Bioinformatics Technology Co., Ltd. with a sequencing depth of $5 \times$ using an Illumina Hiseq 2500 . The raw reads filtered out those corresponding to adapter sequences, those with more than a $10 \% \mathrm{~N}$ content, those less than 10 bases, and selected those with more than a $50 \%$ quality value . All sequencing data were aligned to the Saccharum spontaneum reference genome [11] by BWA software (fast and accurate short read alignment with the Burrows-Wheeler Transform) [12] (PCR duplicates reads were further removed by Samtools (v1.3.167, the Sequence Alignment/Map format and SAMtools) [13]. SNPs were identified among 216 samples by the HaplotypeCaller module of GATK (v3.868) in GVCF mode (The Genome Analysis Toolkit: a MapReduce framework for analyzing next-generation DNA sequencing data) [14]. Then a joint call was performed using GATK GenotypeGVCFs for all 216 samples. SNPs were filtered using the GATK VariantFiltration function with the parameter "QD $<2.0$ | | FS > 60.0| | MQRankSum < - 12.5| | ReadPosRankSum < -8.0| | SOR > 3.0| | MQ < 40.0| | DP > 30| | DP < 3". SNPs with a minimum allele frequency (MAF) $\geq 5 \%$ and a missing rate $\leq 50 \%$ were kept for downstream analysis.

\section{Linkage disequilibrium analysis}

Linkage disequilibrium (LD) $r^{2}$ was calculated using SNPs with MAF > 0.05 and a missing rate $<0.5$ by PLINK (v.1.90b3.42, PLINK: a toolset for whole-genome association and population-based linkage analyses) with the following parameters: -ld-window-r ${ }^{2} 0$-ld-window 99999 -ld-window-kb 500 [15]. The genomewide average $r^{2}$ between two SNPs within $500-k b$ windows was calculated and the distance of LD decay was represented as the physical distance over which the $\mathrm{r}^{2}$ drops to 0.1 .

\section{Genome-Wide Association Study}

SNPs were imputed by the Beagle software with default parameters (A one-penny imputed genome from the next-generation reference panel) [16]. Kinship was analyzed by an emmax-kin module in emmax software with the parameters of $-\mathrm{v}-\mathrm{h}-\mathrm{s}-\mathrm{d} 10$ (Variance component model to account for sample structure in genome-wide association studies) [17]. We used the admixture software (http://software.genetics.ucla.edu/admixture/, v1.3.0) to perform population structure analysis. GWAS was carried out using the Emmax software in a linear mixed model with kinship matrix and population structure (Variance component model to account for sample structure in genome-wide association studies) [18]. The number of independent SNPs was calculated, which used to determine the genome-wide significance cutoff of GWAS. Finally, the significance cutoff of GWAS was $-\log (P) \geq 6$.

\section{Candidate gene for associated SNPs}

We started by merging significant SNPs based on an $r^{2}$ measure of LD $\geq 0.3$. We then defined the confidence intervals as the $250 \mathrm{~kb}$ flanking region around each LD block. Genes located within the confidence interval were classified as candidate genes. Interproscan software (v5.39-77.0) with default parameters was applied to the annotated genes in Saccharum spontaneum with the protein sequences as input files.

\section{Declarations}

\section{Acknowledgements}

We thank Junjian Huang for the sugarcane field management in the base of Institute of Nanfan \& Seed Industry, Guangdong Academy of Sciences (Guangzhou Sugarcane Research Institute).

\section{Authors' contributions}

Qi Yongwen conceived the experiment. Chen Xinglong and Huang zhenghui performed the research, Zhang Xiangbo analyzed the data. Xie Jinfang, Wu Bin, Luo Yiji and Zhu Mingfeng measured the leaf angle and collected the data. Feng Xiaomin helped prepare markers for GWAS. Chen Xinglong drafted the manuscript. All authors read and approved the final manuscript.

\section{Funding}

The work was funded by the special project of Guangdong Academy of Sciences, China (2019GDASYL-0103034); National Key Research and Development Program of China (2018YFD1000503); The National Natural Science Foundation of China (32072027); and the Special Project for Research and Development in Key areas of Guangdong Province (2019B020238001).

\section{Availability of data and materials}

The datasets generated during the current study are available from the corresponding author on reasonable request.

\section{Ethics approval and consent to participate}

Not applicable.

\section{Consent for publication}

Not applicable.

\section{Competing interests}

The authors declare that they have no competing interests. 


\section{References}

1. Dahlquist E. Biomass as Energy Source: Resources, Systems and Applications. London: CRC Press; 2013.

2. Donald CM. The breeding of crop ideotypes. Euphytica. 1968;17(3):385-403.

3. Isidro J, Knox R, Clarke F, Singh A, Somers D. Quantitative genetic analysis and mapping of leaf angle in durum wheat. Planta. 2012;236(6):1713-23.

4. Zhang S, Wang S, Xu Y, Yu C, Shen C, Qian Q, et al. The auxin response factor, Os ARF19, controls rice leaf angles through positively regulating OsGH3-5 and Os BRI1. Plant Cell Environ. 2015;38:638-54.

5. Li C, Li Y, Shi Y, Song Y, Zhang D, Buckler ES, et al. Genetic control of the leaf angle and leaf orientation value as revealed by ultra-high density maps in three connected maize populations. PLoS ONE. 2015;10(3):e0121624.

6. Gouy M, Rousselle Y, Thong CA, Anglade RS, Nibouche CL. Genome wide association mapping of agro-morphological and disease resistance traits in sugarcane. Euphytica. 2015;202:269-84.

7. Banerjee N, Siraree A, Yadav S, Kumar S, Singh J, Kumar S, et al. Marker-trait association study for sucrose and yield contributing traits in sugarcane (Saccharum spp. hybrid). Euphytica. 2015;(1):185-201.

8. Yang X, Todd J, Arundale R, Binder JB, Luo Z, Islam MS, et al. Identifying loci controlling fiber composition in polyploid sugarcane (Saccharum spp.) through genome-wide association study. Ind Crops Prod. 2019;130:598-605. https://doi.org/10.1016/j.indcrop.2019.01.023.

9. Fickett N, Gutierrez A, Verma M, Pontif M, Hale A, Kimbeng C, Baisakh N. Genome-wide association mapping identifies markers associated with cane yield components and sucrose traits in the Louisiana sugarcane core collection. Genomics. 2019;111(6):1794-801.

10. Aljanabi SM, Forget L, Dookun A. An improved and rapid protocol for the isolation of polysaccharide and polyphenol-free sugarcane DNA. Plant Mol Biol Report. 1999;17:281.

11. Zhang J, Zhang X, Tang H, Zhang Q, Hua X, Ma X, et al. Allele-defined genome of the autopolyploid sugarcane Saccharum spontaneum L. Nat. Genet. 2018;50:1565-73.

12. Giannoulatou E, Park SH, Humphreys DT, Ho JWK. Verification and validation of bioinformatics software without a gold standard: a case study of BWA and Bowtie. BMC Bioinformatics. 2014;15:15.

13. Zhao F, Wang Y, Zheng J, Wen Y, Qu M, Kang S, et al. A genome-wide survey of copy number variations reveals an asymmetric evolution of duplicated genes in rice. BMC Biol. 2020;18:73.

14. Bernhardsson C, Wang X, Eklöf H, Ingvarsson PK. Variant Calling Using Whole Genome Resequencing and Sequence Capture for Population and Evolutionary Genomic Inferences in Norway Spruce (Picea Abies). The Spruce Genome. 2020:9-36.

15. Sadowski M, Kraft A, Szalaj P, Wlasnowolski M, Tang Z, Ruan Y, et al. Spatial chromatin architecture alteration by structural variations in human genomes at the population scale. Genome biology. 2019;20(1):148.

16. Khvorykh GV, Khrunin AV. Imputeqc: an R package for assessing imputation quality of genotypes and optimizing imputation parameters. BMC Bioinformatics. 2020;21:304.

17. Li Y, Cao K, Zhu G, Fang W, Chen C, Wang X, et al. Genomic analyses of an extensive collection of wild and cultivated accessions provide new insights into peach breeding history. Genome Biol. 2019;20:36.

18. Pino Del Carpio D, Lozano R, Wolfe MD, Jannink JL. Genome-Wide Association Studies and Heritability Estimation in the Functional Genomics Era. Population Genomics. 2018:361-452.

19. Luo J, Zhang H, Lin Y, Deng Z, Chen R. Relationship between Canopy Structures at Seedling Stage and Yield Characters in Sugarcane. Chinese Journal of Tropical Crops. 2004;25(3):24-8. (in Chinese with English abstract).

20. Luo J, Que Y, Zhang H, Xu L. Seasonal Variation of the Canopy Structure Parameters and Its Correlation with Yield-Related Traits in Sugarcane. The Scientific World Journal. 2013:1-11.

21. Hittalmani S, Huang N, Courtois B, Venuprasad R, Shashidhar HE, Zhuang JY, et al. Identification of QTL for growth-and grain yield-related traits in rice across nine locations of Asia. Theor Appl Genet. 2003;107(4):679-90.

22. Xu F, Sun X, Chen Y, Huang Y, Tong C, Bao J, et al. Rapid Identification of Major QTLs Associated with Rice Grain Weight and Their Utilization. PLoS ONE. 2015;10(3):e0122206.

23. Dubos C, Stracke R, Grotewold E, Weisshaar B, Martin C, Lepiniec L. MYB transcription factors in Arabidopsis. Trends Plant Sci. 2010;15:573-81.

24. Zhang ZB, Zhu J, Gao JF, Wang C, Li H, Li H, et al. Transcription factor At MYB103 is required for anther development by regulating tapetum development, callose dissolution and exine formation in Arabidopsis. Plant J. 2007;52(3):528-38.

25. Shin R, Burch AY, Huppert KA, Tiwari SB, Murphy AS, Guilfoyle TJ, et al. The Arabidopsis transcription factor MYB77 modulates auxin signal transduction. Plant Cell. 2007;19(8):2440-53.

26. Cao WL, Chu RZ, Zhang Y, Luo J, Su YY, Xie LJ, et al. Os JAMyb, a R2R3-type MYB transcription factor, enhanced blast resistance in transgenic rice, Physiological. Molecular Plant Pathology. 2015;92:154-60.

27. Moon J, Candela H, Hake S. The Liguleless narrow mutation affects proximal-distal signaling and leaf growth. Development. 2013;140(2):405-12.

28. Zhang J, Ku LX, Han ZP, Guo SL, Liu HJ, Zhang ZZ, et al. The zmcla4 gene in the qla4-1 qtl controls leaf angle in maize (zea mays I.). J Exp Bot. 2014;65(17):5063-76.

29. Yoshikawa T, Ito M, Sumikura T, Nakayama A, Nishimura T, Kitano H, et al. The rice FISH BONE gene encodes a tryptophan amino transferase,which affects pleiotropic auxin related processes. Plant J. 2014;78(6):927-36. 
30. Miao LJ, Ju PS, Jin H, Jin LE, Hu XY, Je B, et al. Loose Plant Architecture1 (LPA1) determines lamina joint bending by suppressing auxin signalling that interacts with C-22-hydroxylated and 6-deoxo brassinosteroids in rice. J Exp Bot. 2016;6:1883-95.

31. Zhao SQ, Xiang JJ, Xue HW. Studies on the rice LEAF INCLINATION1 (LC1), an IAA-amido synthetase, reveal the effects of auxin in leaf inclination control. Mol Plant. 2013;6(1):174-87.

32. Song YL, You J, Xiong LZ. Characterization of OsIAA1 gene, a member of rice Aux/IAA family involved in auxin and brassinosteroid hormone responses and plant morphogenesis. Plant Mol Biol. 2009;70(3):297-309.

33. Shen CJ, Wang SK, Bai YH, Wu YR, Zhang SN. Functional analysis of the structural domain of ARF proteins in rice (Oryza sativa L.). J Exp Bot. 2010;61(14):3971-81.

34. Ning J, Zhang BC, Wang NL, Zhou YH, Xiong LZ. Increased leaf angle1, a Raf-like MAPKKK that interacts with a nuclear protein family, regulates mechanical tissue formation in the Lamina joint of rice. Plant Cell. 2011;23(12):4334-47.

35. Hopkins L, Parmar S, Bouranis DL, Howarth JR, Hawkesford MJ. Coordinated expression of sulfate uptake and components of the sulfate assimilatory pathway in maize. Plant Biol. 2004;6(4):408-14.

36. Gagne JM, Downes BP, Shiu SH, Durski AM, Vierstra RD. The F-box subunit of the SCF E3 complex is encoded by a diverse superfamily of genes in Arabidopsis. Proc Natl Acad Sci USA. 2002;99(17):11519-24.

37. Qiao H, Chang KN, Yazaki J, Ecker JR. Interplay between ethylene, ETP1/ETP2 F-box proteins, and degradation of EIN2 triggers ethylene responses in Arabidopsis. Genes Dev. 2009;23(4):512-21.

38. Hirano K, Asano K, Tsuji H, Kawamura M, Mori H, Kitano H, et al. Characterization of the Molecular Mechanism Underlying Gibberellin Perception Complex Formation in Rice. The Plant Cell. 2010:2680-2696.

39. Tromas A, Paque S, Stierlé V, Quettier AL, Perrot-Rechenmann C. Auxin-Binding Protein 1 is a negative regulator of the SCF(TIR1/AFB) pathway. Nat Commun. 2013;4:2496.

40. Hammes UZ, Nielsen E, Honaas LA, Taylor CG, Schachtman DP. AtCAT6, a sink-tissue-localized transporter for essential amino acids in Arabidopsis. Plant J. 2010;48(3):414-26.

41. Kunz HH, Husler RE, Fettke J, Herbst K, Schneider A. The role of plastidial glucose-6-phosphate/phosphate translocators in vegetative tissues of Arabidopsis thaliana mutants impaired in starch biosynthesis. Plant Biol. 2010;12:115-28.

42. Dekkera JP, Dekkera EJ. Supramolecular organization of thylakoid membrane proteins in green plants. Biochim Biophys Acta. 2005;1706(1-2):12-39.

\section{Figures}

\section{Image not available with this version}

Figure 1

Leaf angle of different sugarcane genotype at elongation stage.

\section{Image not available with this version}

Figure 2

Frequency distribution of leaf angle in sugarcane different growth stages. A and B indicates +2 and +3 leaf angle in seedling stage respectively (206 accessions); C, D and E indicates $+2,+3$ and +4 leaf angle in elongation stage respectively (203 accessions); F, G and $\mathrm{H}$ indicates $+2,+3$ and +4 leaf angle in mature stage respectively (211 accessions). 


\section{Image not available with this version}

\section{Figure 3}

Pearson's correlation matrix for leaf angle in sugarcane different growth stages. The shaded scale refers to the strenght of correlation. In Pearson's correlation, abs $r=0.5$ to 1 means a greater correlation, abs $r=0.3$ to 0.5 means medium correlation, abs $r=0.1$ to 0.3 means lesser correlation, and abs $r=0$ to 0.1 suggeast no correlation. SS2. and SS3. denotes +2 and +3 leaf of sugarcane seedling stage; ES2. ES3. and ES3. denotes $+2,+3$ and +4 leaf of sugarcne elongation stage; MS2. MS3. and MS4. denotes $+2,+3$, and +4 leaf of sugarcane mature stage.

\section{Image not available with this version}

Figure 4

Population structure of 216 sugarcane core parents and their derived lines. A, Phylogenetic tree of 216 sugarcane core parents and their derived lines, each distinct group were marked with a specific color; B, Subpopulations inferred using STRUCTURE.

\section{Image not available with this version}

Figure 5

Chromosomal locations of the leaf angle loci mapped in sugarcane three growth stages S2 and S3 denotes +2 and +3 leaf of sugarcane seedling stage; E2, E3 and $\mathrm{E} 4$ denotes $+2,+3$ and +4 leaf of sugarcane elongation stage; $M 2, M 3$ and M4 denotes $+2,+3$, and +4 leaf of sugarcane mature stage.

\section{Supplementary Files}

This is a list of supplementary files associated with this preprint. Click to download.

- Attachfiles.rar 